\title{
Cecal Fibromatosis
}

\section{(Desmoid Tumor) Mimicking Periappendicular Abscess: A Case Report}

\author{
Zvi Peled Revital Linder Haim Gilshtein Eli Kakiashvili \\ Yoram Kluger
}

Division of Surgical Oncology, Department of General Surgery, Rambam Health

Care Center, Haifa, Israel

\section{Key Words}

Desmoid tumor · Appendix · Periappendicular abscess · Fibromatosis

\section{Abstract}

A 32-year-old man presented to our department with abdominal pain and fever. In an earlier hospitalization he was diagnosed with periappendicular abscess and treated with antibiotics. Due to fever and 'non-resolution' of the abscess and due to its deep location in the lower abdomen, which excluded percutaneous drainage, we elected to operate the patient. A large mass in the cecum accompanied with an abscess resulted in a right hemicolectomy. The pathological examination revealed a desmoid tumor of the cecum. The patient's recovery was uneventful.

\section{Introduction}

Desmoid tumors are rare non-encapsulated, locally invasive soft tissue tumors of fibrous origin that lack the ability to metastasize, but are very notorious for recurrence. These tumors are also referred to as fibromatosis. Desmoids are characterized histologically by spindle-shaped cells surrounded by and separated from one another by abundant collagen [1]. The tumor can occur in various locations and tissues. Extra-abdominal desmoids occur in the chest wall, back, hip-gluteal region, shoulder girdle, upper arm and thigh. Intra-abdominal desmoids are rare and occur in the pelvis or mesentery [1].

The annual incidence rate of mesenteric desmoid is estimated at 2-5 per million. Familial adenomatous polyposis (FAP) [2] is the most common pathology associated 
with desmoid tumors of the mesentery. A strong association is found with Gardner's syndrome $[2,3]$. The tumors can arise also sporadically. Recognized risk factors are trauma, female sex, estrogen treatment, pregnancy, trisomy 20 and 8, and the presence of the adenomatous polyposis coli germ line mutation. Desmoids represent a major cause of morbidity and mortality in FAP patients [3].

When medically and technically feasible, desmoid tumors are treated by surgical resection aiming at negative margin. Complete resection of the tumor with negative microscopic margins is the standard surgical goal but is often constrained by anatomic boundaries. Extra-abdominal and abdominal wall desmoids are more often resectable than intra-abdominal tumors. Surgery may be difficult and even impossible for intra-abdominal desmoids, particularly when associated with FAP and involving the root of the small bowel mesentery $[4,5]$.

The recurrence rate is high for desmoid tumors at all locations, even following complete resection. The effect of margin status on recurrence rate is unclear. Aggressive resection with negative margins has been reported to result in recurrence rates of $16-39 \%[4]$.

We describe a case of a very rare desmoid tumor of the wall of the cecum that resulted in perforation, subsequent abscess formation, and misdiagnosis and treatment as a periappendicular abscess.

\section{Case Report}

A 32-year-old previously healthy male presented to another hospital with a 7-day history of abdominal pain located in the right lower quadrant. He was afebrile. Abdominal examination revealed local tenderness. Rebound tenderness and guarding were noticed in the right lower abdominal quadrant. Laboratory tests revealed a white blood cell count of 13,700 . The patient was admitted to the surgical ward and treated with antibiotics. He was discharged 4 days after an uneventful course. He was advised to continue oral antibiotics and to undergo a colonoscopy 6 weeks following the discharge. Three weeks after hospital discharge the patient was referred to our emergency department due to fever, chills and recurrent right lower quadrant abdominal pain. On physical examination local right abdominal tenderness was detected. Laboratory tests revealed a white cell count of 11,200. Due to the local tenderness and the previous diagnosis of periappendicular abscess, repeat abdominal tomography was performed in order to evaluate the accessibility of the abscess to percutaneous drainage. The abdominal tomography showed a large mass originating in the cecum with abscess formation (fig. 1). Lymphadenopathy was noticed in the large and small bowel mesentery. Due to the suspicion of a tumor that resulted in perforation and abscess formation, which did not resolve with antibiotic treatment, we elected to operate on the patient.

At laparotomy a large submucosal mass obstructing the appendix orifice was noted. The appendix was dilated. A large abscess was attached to the lateral wall of the cecum along the appendix. This organized abscess had a very thick wall. A right hemicolectomy was carried out. The patient was discharged after an uneventful 6-day post-operative course.

The pathological report revealed the mass to be an 8-cm large fibromatosis tumor (desmoids). The tumor cells stained for $\beta$-catenin. The appendix was normal. Twenty-four lymph nodes harvested at surgery showed reactive changes only. 


\section{Discussion}

Mesenteric desmoid tumors are rare, with an estimated annual incidence of 2-5 per million [1]. The tumors can arise sporadically or in association with FAP.

Fibromatosis are benign fibrous neoplasms originating from the musculoaponeurotic structures throughout the body. These tumors are locally aggressive and may compress surrounding structures. Their treatment consists of surgical removal with negative margins [4]. The case described represents a cecal wall desmoid tumor in a patient with no family or personal history of FAP or Gardner's syndrome, and no personal history of trauma or previous surgeries.

Intra-abdominal desmoids are usually asymptomatic until their size causes compression of surrounding viscera. This compression can lead to intestinal obstruction, ischemic bowel secondary to vascular compression and hydronephrosis due to ureteric compression.

Histologically, these tumors are composed of collagen that surrounds poorly circumscribed spindle cells. The cytoplasm is pale with regular nuclei, and with neither mitoses nor giant cells. Macrophages, giant cells and lymphocytes are present in the periphery [2].

Complete surgical excision may be the only effective cure for desmoids. However, since complete excision is often impossible, adjuvant treatments have been employed with various degrees of success [3]. The optimal treatment has not yet been established and, in many cases, a multidisciplinary approach including surgery, chemotherapy, and radiation therapy has been employed [5].

\section{Conclusion}

Desmoid tumors originate from musculoaponeurotic structures and can be extra- or intra-abdominal. Emergency presentation is not common, but is usually secondary to a mass effect. In rare cases, such as the one we present, the site of the tumor can be the wall of the involved bowel. Periappendicular abscess is not uncommon. Surgeons caring for patients diagnosed with this pathology should be aware of tumor etiology and exclude remote diagnosis resulting in abscess formation in the appendix or its surroundings. CT and MRI are the imaging modalities of choice. Diverse clinical course and atypical clinical behavior should raise the suspicion of rare clinical entities. 


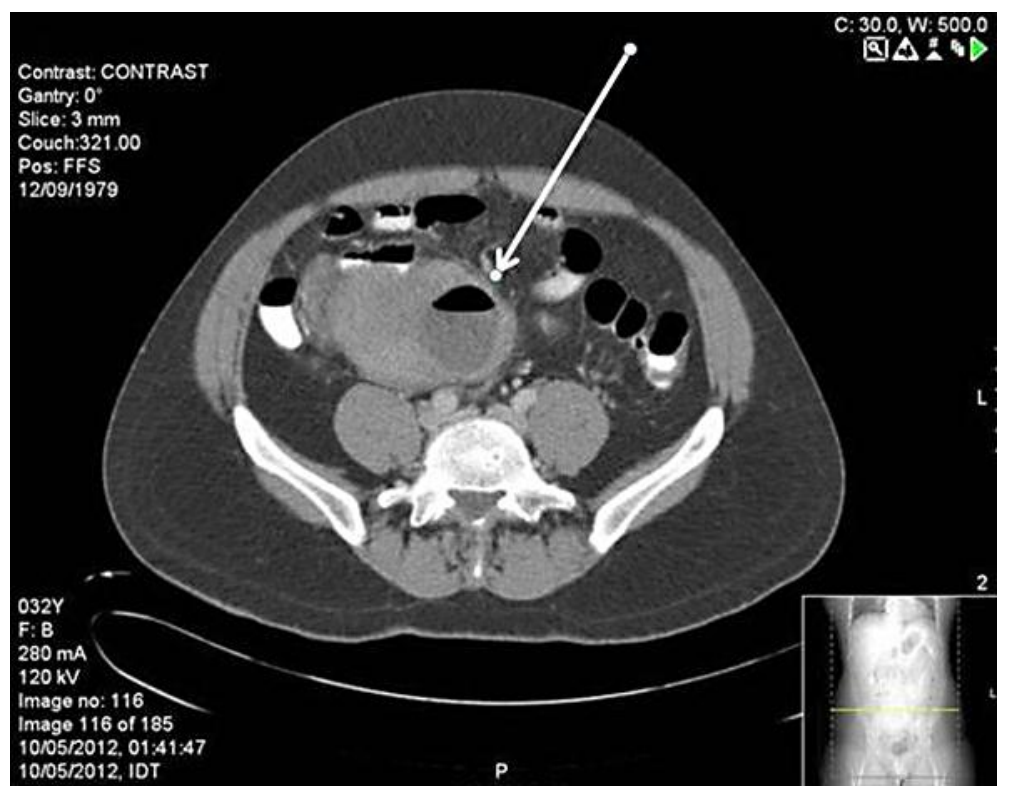

Fig. 1. Abdominal tomography image showing the large mass originating in the cecum with abscess formation.

\section{References}

-1 Shinagare AB, Ramaiya NH, Jagannathan JP, et al: A to Z of desmoid tumors. AJR Am J Roentgenol 2011;197:1008-1014.

2 Sinha A, Tekkis PP, Gibbons DC, et al: Risk factors predicting desmoid occurrence in patients with familial adenomatous polyposis: a meta-analysis. Colorectal Dis 2011;13:1222-1229.

-3 Gurbuz AK, Giardello FM, Petersen GM, et al: Desmoid tumors in familial adenomatous polyposis. Gut 1994;35:377-381.

4 Nuyttens JJ, Rust PF, Thomas CR Jr, Turrisi AT 3rd: Surgery versus radiation therapy for patients with aggressive fibromatosis or desmoid tumors: a comparative review of 22 articles. Cancer 2000;88: 1517-1523.

5 Lahat G, Nachmany I, Itzkowitz E, et al: Surgery for sporadic abdominal desmoid tumor: is low/no recurrence an achievable goal? Isr Med Assoc J 2009;6:398-402. 